\title{
Principais técnicas instrumentais de caracterização de material particulado na atmosfera
}

\author{
Galvão, E. S. ${ }^{1 *}$; Feroni, R. C. ${ }^{1,2} ;$ Andreão, W. L. ${ }^{1}$; Orlando, M. T. D. ${ }^{3} ;$ Passos, C. A. C. ${ }^{3}$ \\ ${ }^{1}$ Programa de Pós-Graduação em Engenharia Ambiental, Universidade Federal do Espírito Santo, Vitória, ES, Brasil. \\ ${ }^{2}$ Departamento Regional da Bahia, Centro Integrado de Manufatura e Tecnologia, Salvador, BA, Brasil. \\ ${ }^{3}$ Programa de Pós-Graduação em Engenharia Mecânica, Universidade Federal do Espírito Santo, Vitória, ES, Brasil. \\ *e-mail: elsongalvao@gmail.com
}

\begin{abstract}
Resumo
O material particulado (MP) presente na atmosfera de grandes centros urbanos provém de origem natural e antropogênica. A massa e composição química dessas partículas estão relacionadas a um grande número de casos de internação hospitalar e morte, causados principalmente por doenças respiratórias e cardiovasculares. Estudos recentes têm demonstrado a importância da caracterização química e morfológica do MP presente na atmosfera como forma de identificar as potenciais fontes de emissão dessas partículas, visando o controle e melhoria continua da qualidade do ar, além de fornecer subsídios a pesquisas relacionadas à epidemiologia e toxicologia das partículas. Diversas técnicas de caracterização de partículas são descritas na literatura, como espectrometria, difratometria, microscopia entre outras. Contudo, poucos autores abordam as vantagens e limitações das técnicas quanto sua aplicação na identificação das fontes de emissão. O presente trabalho traz uma abordagem prática das principais técnicas instrumentais de análise química e morfológica de MP, explicitando suas vantagens e limitações na associação da identidade das partículas com potenciais fontes de emissão.
\end{abstract}

\begin{abstract}
Particulate matter (PM) present in urbanized areas consists of both natural and human activities. Mass and composition of particles are related to a large number of hospital admission and death caused by respiratory and cardiovascular issues. Recent studies have shown that is primordial the chemical and morphological characterization of the PM as a way to identify the potential sources of these pollutants, aiming the mitigation and the continuous improvement of air quality, as well as provide information about the particles for toxicological and epidemiological researches. Several technics are reported in literature for particle characterization. However, few authors discuss the advantages and limitations of different techniques as their application in identifying the PM sources. This work presents a practical approach of the main instrumental analytical techniques applied to the study of air pollution focusing on its advantages and limitations in pool identity particle with potential emission sources.
\end{abstract}

Keywords (Palavras chaves): Particulate matter (Material particulado), Emission sources (Fontes de emissão) Analytical techniques (Técnicas de análise).

\section{Introdução}

Nas últimas décadas especial atenção tem sido direcionada por parte da comunidade científica, de órgãos ambientais e da sociedade organizada aos problemas envolvendo a presença de material particulado (MP) quer seja em suspensão, quer seja depositado no meio ambiente. Diversos estudos epidemiológicos demostram a associação entre o MP na atmosfera e o aumento das taxas de mortalidade em humanos, especialmente idosos e crianças $[1,2,3]$.

O MP presente na atmosfera é uma mistura de elementos metálicos, carbono, amônia, nitratos, 
sulfatos, minerais, elementos traços e água [4]. O MP é constituído por partículas sólidas ou líquidas de tamanho, forma e composição variada de acordo com sua fonte de origem e transformações durante seu transporte na atmosfera [5]. O diâmetro e densidade das partículas determinam quão longe das fontes as partículas são transportadas e quão profundas são depositadas no sistema respiratório [6]. Contudo, os efeitos à saúde da população exposta ao MP podem estar mais fortemente relacionados à sua composição do que à sua massa e tamanho [7]. Assim, uma relação entre as características químicas e morfológicas do MP são determinadas por suas fontes emissoras [8] e, portanto, podem ser utilizados para inferir sua origem [9] com a finalidade de prover informações a respeito da responsabilidade e tomada de decisões em relação à mitigação dos impactos ambientais pelos órgãos ambientais competentes. Desta forma, neste trabalho os autores desejam mostrar as principais técnicas de análise de tamanho de partículas...

\subsection{Monitoramento das partículas}

O método de coleta do MP deve considerar o objetivo de cada análise. Para cada faixa de tamanho de MP há um método de referência [10]. As faixas granulométricas podem ser separadas com o auxilio de um impactador inercial, conforme esquema resumido na Figura 1.

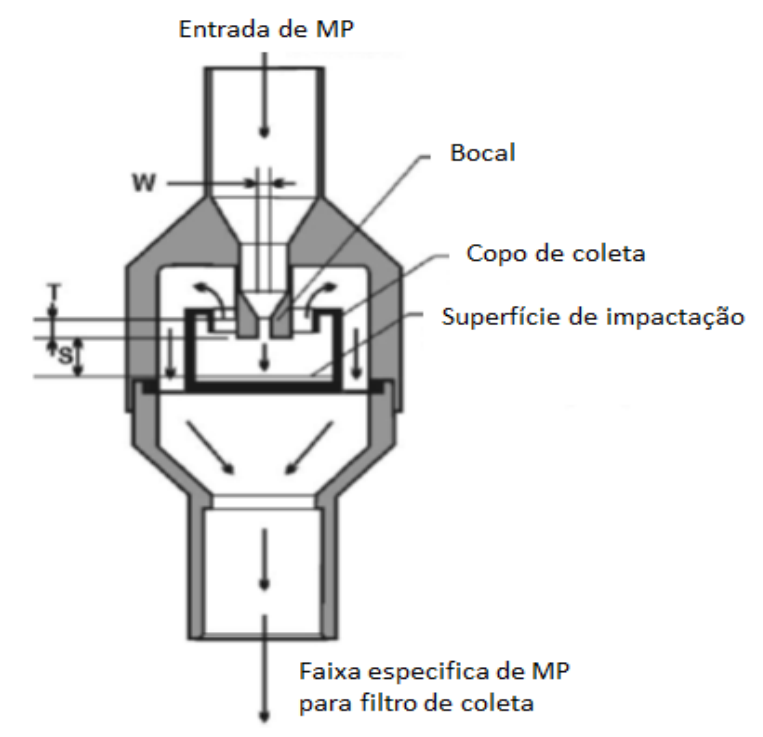

Figura 1. Esquema de um impactador WINS [11].
Diversos filtros, ou membranas, estão disponíveis no mercado, porém, a escolha do material que as compõe deve levar em consideração os elementos a serem analisados. Membranas de Teflon são adequadas para análise elementar de metais. Membranas de Nylon com denuder para nitratos e sulfatos, e membranas de quartzo para análises de carbono [11].

\subsection{Análise elementar de partículas}

A exceção de elementos como $\mathrm{H}, \mathrm{O}, \mathrm{C}$ e N , as técnicas a seguir são capazes de caracterizar elementos desde o sódio ao urânio, diferindo apenas a respeito dos limites de detecção, preparação de amostra e custo financeiro [12]. Dentre as principais técnicas de análise elementar destacam-se:

(1) Fluorescência de raios-X (XRF) e suas variantes (EDXRF, WDXRF e TXRF);

(2) Emissão de raios-X por indução de prótons (PIXE);

(3) Análise por ativação de nêutrons (INAA);

(4) Espectrometria de absorção atômica (AAS);

(5) Espectrometria de emissão atômica por plasma acoplado indutivamente (ICP-AES);

(6) Espectrometria de massa por plasma acoplado indutivamente (ICP-MS).

Além da aplicação na caracterização elementar, as técnicas AAS, ICP-AES e ICP-MS também são apropriadas a caracterização de íons simples extraídos em soluções.

Os íons poliatômicos devem ser quantificados por outras técnicas, entre tais:

(7) Cromatografia iônica (IC);

(8) Colorimetria automatizada (AC).

Entre as técnicas térmicas para medição de carbono orgânico (OC) e carbono elementar (EC) destacam-se:

(9) Refletância termo/ótica (TOR); 
(10) Transmissão termo/ótica (TOT);

(11) Oxidação térmica por manganês (TMO).

Diversos autores têm escolhido as técnicas de XRF (1) e PIXE (2) para a caracterização química de MP em filtros [13-15] devido sua facilidade operacional, possibilitando análise multielementar, não destrutiva, além de não exigir preparação das amostras. As técnicas (1) e (2) baseiam-se na produção e detecção de raios $X$ característicos emitidos pelos elementos quando irradiados com elétrons, íons, raios $\mathrm{X}$, ou gama, com energias específicas.

A técnica PIXE, além das vantagens já mencionadas, é capaz de determinar elementos de interesse em níveis de ppb [16]. Essas duas técnicas têm sido amplamente utilizadas em estudos de caracterização de MP com o objetivo de identificar suas fontes de emissão $[13,17]$.

A técnica INAA se baseia no bombardeamento de nêutrons sobre a amostra transformando diversos elementos em isótopos radioativos que emitem diferentes energias de raios gama, os quantificando de acordo com a intensidade. É possível utilizar uma combinação de técnicas XRF e INAA no aprimoramento das medições. Cada técnica possui melhor sensibilidade a um conjunto de elementos, porém, em termos de limite de detecção, ambas se equivalem. A INAA possui a limitação de no processo tornar os filtros frágeis e radioativos, limitando sua reutilização, além disso, o tempo de análise pode durar algumas horas [11].

A técnica de AAS (4) se baseia na absorção de luz a um comprimento de onda específico do espectro visível por cada elemento e comparação com padrões de referência para a quantificação dos elementos. Esta técnica é conveniente na complementação de outros métodos (XRF, PIXE), principalmente na quantificação de espécies como $\mathrm{Na}, \mathrm{K}$ e $\mathrm{Mg}$, contudo, a AAS requer análises individuais para cada elemento, aumentando o tempo de análise e interferência do operador. As técnicas ICP (5) e (6) baseiam-se na ionização, excitação e relaxamento das partículas da amostra por um plasma induzido, se diferenciando no método de detecção entre (5) e (6). O limite de detecção da técnica ICP-MS pode chegar a ppq. A grande desvantagem dessas técnicas está na preparação das amostras, devendo estar na fase liquida, portanto, para análises de MP as amostras devem ser digeridas, geralmente em meio ácido, causando a destruição da amostra [11, 12]. Além disso, Amostras de MP podem ter composições complexas podendo apresentar resíduos insolúveis e co-precipitação. A técnica de ICPMS foi utilizada na caracterização de isótopos de chumbo como forma de identificar fontes de emissão de poluentes metálicos em MP a partir de operações de mineração [18].

Devido a processos físicos e químicos na atmosfera, poluentes gasosos como $\mathrm{Cl}_{2}, \mathrm{NH}_{3}, \mathrm{NO}_{2}$ e $\mathrm{SO}_{2}$ sofrem reações os transformando em espécies iônicas como $\mathrm{Cl}^{-}, \mathrm{NH}_{4}^{+}, \mathrm{NO}_{3}^{-}$e $\mathrm{SO}_{4}^{-2}$ que aderem ao MP suspenso na atmosfera, fazendo parte de sua constituição [19]. Para a caracterização de tais íons, comumente são utilizadas as técnicas de IC (7) e AC (8). Na técnica de IC, a amostra extraída em fase liquida passa por uma coluna para eluição/retenção dos íons, causando uma separação das espécies iônicas no tempo, seguida de sua quantificação, geralmente, por meio de um detector de eletrocondutividade.

Para espécies catiônicas, como o $\mathrm{NH}_{4}{ }^{+}$, é mais comum o uso da AC. A colorimetria apresenta a vantagem de pouca manutenção e baixo custo. Porém, a pesar da IC exigir maior habilidade do operador na interpretação dos cromatogramas, a sensibilidade e limite de detecção desta técnica são mais bem apropriadas quando as amostras possuem grande variedade de íons [11], como o caso de partículas atmosféricas.

Os resultados obtidos por meio das técnicas descritas de (1) à (11) são comumente utilizados como dados de entrada de modelos estatísticos, ou modelos receptores, para a identificação e quantificação da contribuição de fontes de emissão [20]. Contudo, os modelos são bastante sensíveis à colinearidade entre fontes, ou seja, a similaridade entre a composição química das fontes. A colinearidade afeta a predição de classes, resultando em contribuições de fontes incorretas, relacionando duas ou mais fontes a um 
único fator. Portanto, apenas a caracterização química do MP não é capaz de prover informações a respeito das fontes de emissão quando há fontes similares em uma região. Uma forma de minimizar este efeito está na atribuição de marcadores específicos para as fontes com alta colinearidade [21].

\subsection{Análise morfológica e estrutural de partículas}

As técnicas (12) microscopia eletrônica de varredura (MEV) e (13) difratometria de raios-X (DRX) promovem importantes informações a respeito da mineralogia $e$ morfologia de partículas que podem vir a servir como marcadores específicos que ligam a história dessa partícula a uma fonte específica [22-24] e, portanto, minimizar os efeitos de colinearidade apresentado pelos modelos receptores.

A técnica MEV (12) consiste em utilizar um feixe de elétrons para explorar a superfície da amostra e transmitir o sinal do detector a uma tela catódica gerando um sinal de imagem resultante da interação do feixe incidente com a superfície da amostra. O sinal recolhido pelo detector é utilizado para modular o brilho do monitor, permitindo a observação das características morfológicas do material [25]. O MEV também pode ser acoplado a um EDX (MEV-EDX) o qual possibilita a determinação da composição qualitativa e semi-quantitativas.

A técnica DRX (13) baseia-se na medida da difração de raios-X monocromático causado pela rede cristalina. As técnicas MEV e DRX em conjunto podem promover informações a respeito da distribuição de tamanho, forma, angularidades, textura de superfície e fases cristalinas do MP que podem contribuir na identificação de um marcador específico de fonte [26]. As técnicas descritas também possuem a vantagem de ser não destrutivas, contudo, na DRX a presença de materiais amorfos pode modificar 0 background dos difratogramas quando em quantidades significativas desses materiais. Partículas com diâmetro inferior a 5 $\mu \mathrm{m}$ podem levar a formação de fases amorfas [27], e considerando a complexidade na composição e granulometria das partículas na atmosfera, tal hipótese deve ser considerada. Uma das vantagens da utilização do MEV-EDS está na rapidez e facilidade na preparação das amostras, que depende do objetivo da pesquisa, contudo seu custo de aquisição e manutenção é relativamente alto.

\section{Conclusão}

Diversas técnicas estão disponíveis para a caracterização química e morfológica de partículas na atmosfera. Sua escolha deve se basear em um conhecimento prévio das partículas e de fontes potenciais, no intuito de determinar a origem das partículas, tomando o máximo de informações que distinguem características específicas que possam ser relacionadas a uma única fonte. O conhecimento do principio e limitações das técnicas utilizadas na caracterização do MP pode reforçar a qualidade dos resultados obtidos e minimizar tomadas de decisões equivocadas.

\section{Agradecimentos}

Os autores agradecem a CAPES e CIMATEC pelo apoio financeiro.

\section{Referências}

[1] DOCKERY, D.W.;POPE, C.A. Annu. Rev. Public Health.15:107-32, 1994.

[2] WHO - World Health Organization. Global Update 2005. Summary of Risk Assessment. Geneva, 2006.

[3] SALDIVA, P.H.N. et al. Rev. Saúde Pública; 38(6): 751-7, 2004.

[4] HUEGLIN et al. Atmos. Environ. 39:637-651, 2005.

[5] FENG, X. et al. J Atmos Chem., 64:37-51, 2009.

[6] HOLGATE, S.T. et al. Air Pollution and Health, Academic Press, 1999.

[7] GHIO, A.J; DEVLIN, R.B. Am J Respir Crit Care Med 164(4):704-708, 2001.

[8] SLEZAKOVA, K. et al. J Atmos Chem. 60:221-236, 2008.

[9] BERNABE, J.M. et al. Atmos. Environ. 39: 6777-6789, 2005.

[10] US EPA, 1996. Air quality criteria for particulate matter, report no. EPA/600/P-95/001abcF. 
[11] WIILSON, W.E. et al. Chemos. 49:1009-1043, 2002.

[12] CHOW, J.C. J Air Waste Manag Assoc. 45:320-82, 1995.

[13] VINEYARD, M.F. et al. Nucl. Instrum. Methods Phys. Res., B Beam Interact. Mater. Atoms, 350:77-80, 2015.

[14] REYES-HERRERA, J. et al. Microchemical Journal, 120:0-44, 2015.

[15] CHENG, Y. et al. Particuology, 18:96-104, 2015.

[16] JOHANSSON, S.A.E. et al. Particle-Induced X-Ray Emission Spectrometry (PIXE). Wiley-Interscience, New York, 1995.

[17] DÍAZ, R.V. et al. Nucl. Instrum. Methods Phys. Res., B Beam Interact. Mater. Atoms. 318:135-138, 2014.

[18] FÉLIX, O.I. et al. Chemos. 122:219-226, 2015.

[19] FINLAYSON-PITTS, B. J. e PITTS, J. N. Jr. Chemistry of the Upper and Lower Atmosphere. Theory, Experiments, and Applications. WileyInterscience publication, Wiley, New York, 1999.

[20] WATSON, J.G.; et al. Chemos. 49:1093-1136, 2002a.

[21] SHI, G. et al. Aerosol Air Qual. Res. 14:2040-2050, 2014.

[22] HE, C. et al. J. Geogr. 107:1-9, 2007.

[23] AKRAM, W. et al. Environ Monit Assess. 186:8587-8598, 2014.

[24] CONNER, T.L.; WILLIAMS, R.W. Atmos. Environ.38:5305-5310, 2004.

[25] GOLDSTEIN, J.I.; NEWBURY, D.E.; ECHLIN, P.; JOY, D.C.; ROMIG Jr, A.D.; LYMAN, C.E.; FIORI, C.; LIFSHIN, E. Scanning Electron Microscopy and X-Ray Microanalisys: A Text for Biologists, Materials cientists, and Geologists, New York: Plenum Press, 1992.

[26] AVILA, A. et al. J. Geophys. Res. 102:2197721996, 1997.

[27] FORMOSO, M.L.L. Difratometria de Raios X. Técnicas Analíticas Instrumentais Aplicadas à Geologia, São Paulo: Edgard Blücher Ltda. 1984. 